\title{
Modification and Dynamics inside Diamond by Femtosecond Laser Double-Pulse Irradiation
}

\author{
Masaaki Sakakura ${ }^{1}$, Takuro Okada², Vibhav Bharadwaj ${ }^{3}$, Belén Sotillo ${ }^{3}$, Shane M. Eaton ${ }^{3}$, Roberta Ramponi ${ }^{3}$, \\ Ali Serpengüzel ${ }^{4}$, Ulaş S. Gökay ${ }^{4}$, Yasuhiko Shimotsuma ${ }^{2}$ and Kiyotaka Miura ${ }^{2}$ \\ ${ }^{1}$ Office of Society-Academia Collaboration for Innovation, Kyoto Univ., Kyoto, 615-8245, Japan. \\ E-mail: msakakura@saci.kyoto-u.ac.jp \\ ${ }^{2}$ Dept. of Material Chemistry, Graduate School of Eng., Kyoto Univ., Kyoto, Japan. \\ ${ }^{3}$ Istituto di Fotonica e Nanotecnologie (IFN) - CNR, and Dipartimento di Fisica, Politecnico \\ di Milano, Milano, Italy. \\ ${ }^{4}$ Microphotonics Research Laboratory, Department of Physics, Koç University, Istanbul, Turkey
}

\begin{abstract}
We investigated modifications and the temporal evolution of the morphology in the photoexcited region inside diamond single crystal after photoexcitation by tightly focused fs laser pulses. We found that double-pulse irradiation with 200-400 fs laser pulses was able to induce a permanent modification in the photoexcited region, while single-pulse irradiation with 100-300 fs laser pulses was not. The modification by double-pulse irradiation became larger for a longer time-difference between the two pulses in the range from 1 ps to 10 ps. The morphology change observed by a pump-probe optical microscope showed that the photoexcited region by double-pulse irradiation was smaller than by single-pulse irradiation, even for the same total pulse energy, with the amplitudes of the laser induced-stress waves being the same. This observation suggests that the photoinduced plasmas by double-pulse irradiation was localized in a smaller region than that by singlepulse irradiation, and the difference in plasma distribution could be the origin of the permanent modification.
\end{abstract}

DOI: 10.2961/jlmn.2016.03.0012

Keywords: femtosecond laser, diamond, bulk processing, dynamics, crystal

\section{Introduction}

Diamond is an important material not only as a jewel but also as a material for electronics and photonics. It is because diamond has high chemical stability, high refractive index, high phonon conductivity and large optical band gap. In particular, diamond has attracted great interest as a platform for quantum information because it has nitrogen vacancy (NV) centers, which can be exploited as quantum bits since the electron spin at the NV center has a long coherence time, and be controlled with light [1, 2]. In many cases, quantum states are read out or manipulated using a confocal optical microscope. For realization of more compact and integrated diamond-based photonic devices, it is essential to make optical waveguides inside diamond. The key method of writing optical waveguides inside single crystals is femtosecond (fs) laser direct writing (fs-LDW) $[3,4]$. So far, waveguides have been written inside various single crystals by producing a compressive region between two closely-spaced damage lines using fs-LDW. Therefore, in the case of waveguide writing inside diamond, two continuous modification lines should be written by inducing phase transformation, such as diamond-to-graphite, spaceselectively by fs-LDW.

Several research groups have reported space-selective phase transformation inside a diamond by fs-LDW and its applications [5-12]. Kononenko et al. have conduced comprehensive studies on fs laser-induced graphitization inside diamond. Based on their studies, they produced diamondgraphite based detectors for Gamma ray or beta-particles and photonics crystals for infrared light $[6,9]$. Booth et al. have compensated a large spherical aberration in fs-LDW inside diamond using adaptive optics system and succeed to fabricate micrometer-diameter graphite lines inside diamond [10, 11]. Some of the authors of this paper (Shimotsuma et al.) have demonstrated the fabrication of a wire-grid polarization filter for $\mathrm{THz}$ electron magnetic wave by writing graphite lines periodically inside diamond using fs-LDW [12]. Writing of graphite lines in polymer materials was also demonstrated in their study [13]. However in all the studies of fs-LDW inside diamond, the irradiation with a more than 10 pulses was necessary to induce a modification inside diamond [5-12]. For controlling the properties of waveguides inside diamond written by fsDLW, it is necessary to find the optimal laser irradiation conditions that can induce modifications inside diamond by a single shot and with the lowest pulse energy possible.

In this paper, we investigated the method to induce a permanent modification inside single crystal diamond by focused fs laser pulses. We found that no permanent modification appeared even after more than 100 shots of fs laser pulses (pulse durations was 300 fs or shorter), even though the photoexcitation was clearly observed by the pumpprobe transmission microscopy. On the other hand, we discovered that permanent modifications were possible with a single shot using a double-pulse irradiation, when the pulse duration was between 200 fs and 400 fs. In addition, the dynamics after photoexcitation was measured by the observation of transmission and phase change using a pumpprobe transmission and interference microscopy. The dynamics observed through the microscopy includes plasma 
formation, stress wave generation and heat dissipation. Based on the observation of these dynamics, the differences between single and double-pulse fs laser irradiation in diamond are discussed.

\section{Experimental \\ 2.1 Double-pulse irradiation and pump-probe transmission microscope}

Figure 1 shows a schematic illustration of double-pulse irradiation inside a single crystal diamond with a pumpprobe microscope. Two temporally separated fs laser pulses were focused inside a diamond to induce nonlinear photoexcitation at the focus (Fig. 1 (a)). Figure 1 (b) is the optical setup for the double-pulse irradiation and the pumpprobe microscope. The fs laser pulse was generated from a Ti-sapphire fs laser with regenerative amplifier (Coherent; Mira-Legend, the central wavelength was $800 \mathrm{~nm}$, transform-limited pulse duration was 120 fs.). The doublepulses were produced by a Mach-Zehnder interferometertype optical path with an optical delay line (enclosed by a broken line in Fig. 1 (b)). The temporal-difference between the two pulses was controlled by the optical delay line, and the zero-time difference was confirmed by a single shot autocorrelator. We call the temporal difference between the two pulses "pulse delay" and denote it as $\Delta t_{d}$. The doublepulses were expanded by a telescope so that the beam width was as large as the inlet of an objective lens (Nikon, LU Plan 50×, NA $=0.8, f=4 \mathrm{~mm}$ ), and focused inside the diamond single crystal with the objective lens. The diameter of the laser pulse before the objective lens was $8 \mathrm{~mm}$. The estimated spot diameter at the focus was $0.6 \mu \mathrm{m}$, but it would be larger inside diamond because of the spherical aberration by a high refractive index of diamond [10]. The focus of the fs laser pulse was about $50 \mu \mathrm{m}$ deep below the surface of the diamond and the incident of the laser pulses was normal to the (100) plane of the diamond.

In the pump-probe microscope, the second harmonic (SHG) of a fs laser pulse, which had been generated by passing the fs laser pulses through a $\beta-\mathrm{BaB}_{2} \mathrm{O}_{4}(\mathrm{BBO})$ crystal, was used as the probe pulse. The probe pulse, which had been delayed by an optical delay line, was transmitted through the photoexcited region, and the transmitted light expanded by the objective lens was imaged on a charge coupled device (CCD) camera.

The sample was a polished chemical vapour deposition (CVD) single crystal diamond purchased from MB Optics (type II, optical grade with nitrogen impurities $100 \mathrm{ppb}$ ). The size of the sample was $7.5 \mathrm{~mm} \times 7.5 \mathrm{~mm} \times 0.5 \mathrm{~mm}$.

\subsection{Phase imaging in the pump-probe microscope with a Michelson interferometer and the analysis}

To obtain the phase distribution change in the photoexcited region, the interference pattern of a probe pulse was observed using a Michelson interferometer, which was placed before the CCD camera (Fig. 1 (c)). This method is the same as that by Hayasaki et al [14]. In the Michelson interferometer, the probe beam was split into two (P1 and $\mathrm{P} 2$ ), and two identical beams were overlapped on the CCD camera to produce an interference pattern. If the electric fields of two probe beams are written by $E_{1}(x, y)$ and $E_{2}(x, y)$, the interference pattern, $I(x, y)$, can be written by

$$
I(x, y)=\left|E_{1}(x, y)+E_{2}(x, y)\right|^{2}
$$

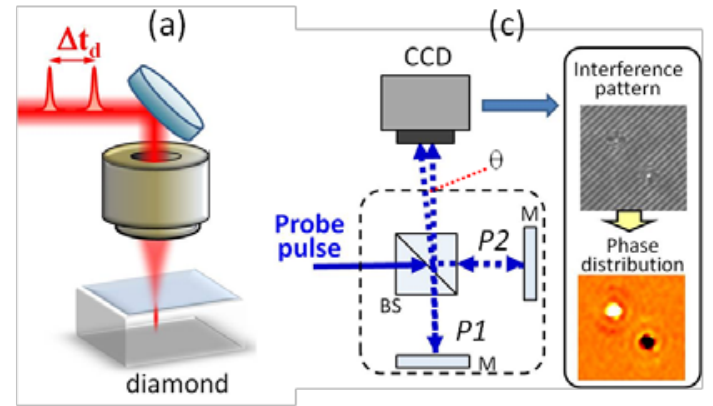

(b)

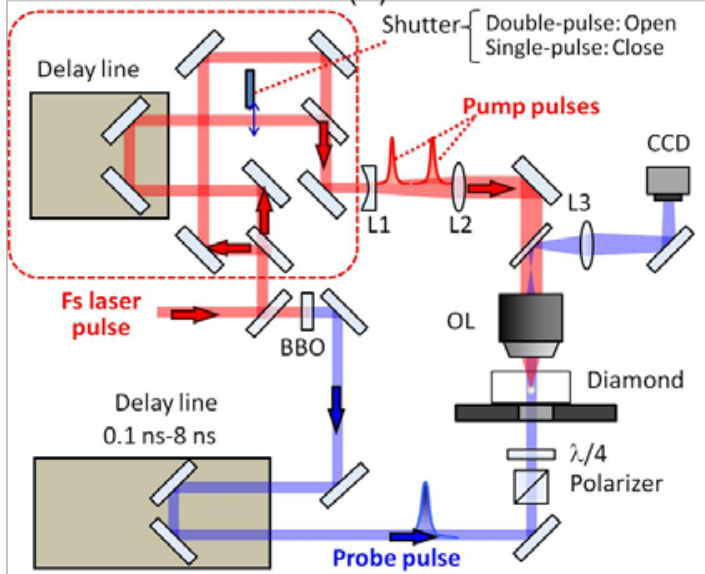

Fig. 1 (a) Double-pulse laser irradiation inside diamond. (b) Experimental setup for double pulse laser irradiation with a pump-probe microscope. L1, L2 and L3 are lenses having focal lengths of $-100 \mathrm{~mm}, 250 \mathrm{~mm}$, and $400 \mathrm{~mm}$, respectively. OL is an objective lens ( $0.8 \mathrm{NA}, f=4 \mathrm{~mm}$ ) and $\lambda / 4$ is a quarter waveplate for the probe pulse. (c) Michelson interferometer for imaging of phase distribution of the probe beam.

When the phase distribution of the probe beam on the CCD is expressed by $\phi(x, y)$, the electric field of the $\mathrm{P} 1, E_{1}(\mathrm{x}, \mathrm{y})$, can be written by

$$
E_{1}(x, y)=A_{1}(x, y) * \exp [i\{\boldsymbol{k} \cdot \boldsymbol{r}+\phi(x, y)\}]
$$

where $\boldsymbol{k}$ is the wavevector of the probe beam, $A_{1}(x, y)$ is the amplitude of the probe beam, $\mathrm{P} 1$, and it is assumed that the $\mathrm{P} 1$ was incident normal to the CCD. If the angle between incidents of two probe beams is $\theta$, the electric field of $\mathrm{P} 2$, $\mathrm{E}_{2}(\mathrm{x}, \mathrm{y})$, can be written by

$$
E_{2}(x, y)=A_{1}\left(x^{\prime}, y\right) * \exp \left[i\left\{\boldsymbol{k}^{\prime} \cdot \boldsymbol{r}+\phi\left(x^{\prime}, y\right)\right\}\right]
$$

where $\boldsymbol{k}^{\prime}$ is the wavevector and $\boldsymbol{k}^{\prime} \cdot \boldsymbol{k}=|\boldsymbol{k}|^{2} \cos \theta$. and $x^{\prime}=x \sin \theta$ $-x_{0}$, and $x_{0}$, is the lateral displacement of $\mathrm{P} 2$ relative to $\mathrm{P} 1$ on the CCD. Substitution of eqs (2) and (3) into eq. (1) gives

$$
\begin{aligned}
& I(x, y)=\left|A_{1}(x, y)\right|^{2}+\left|A_{2}(x, y)\right|^{2} \\
& +A_{1}(x, y) * A_{2}\left(x^{\prime}, y\right) * \cos \left[\phi(x, y)-\phi\left(x^{\prime}, y\right)-|\boldsymbol{k}| x \sin \theta\right]
\end{aligned}
$$

The third term on the right side makes an interference pattern. We obtained $\phi(x, y)-\phi\left(x^{\prime}, y\right)$ in the interference term based on the Fourier transform method, proposed by Takeda et al [15]. Because $x$ and $x^{\prime}$ are spatially separated, the phase distribution in the photoexcited region can be obtained.

\section{Results}

\subsection{Irradiation with single pulse-trains}

Figure 2(a) shows a pump-probe optical microscope image at 1 ps after single-pulse irradiation with a focused 300 fs laser pulse having $2 \mu \mathrm{J}$ pulse energy and that long after 


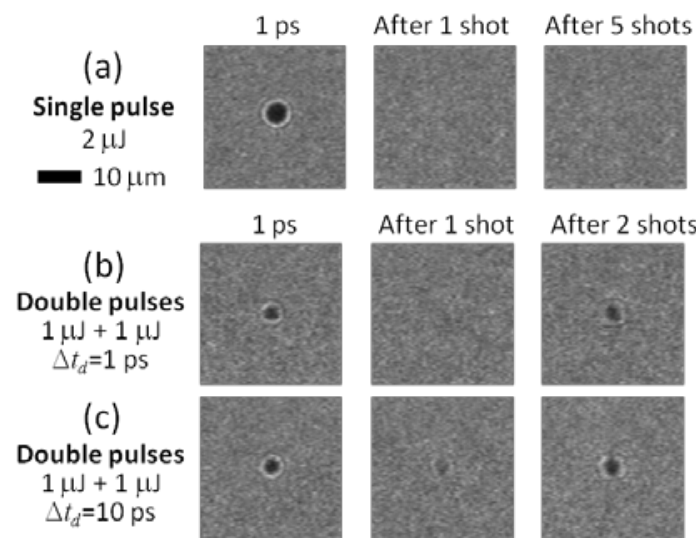

Fig. 2 Pump-probe microscope images of fs laser-induced modifications inside diamond. The transient images in (a)(c) show photoexcited states at 1 ps after the laser irradiation, while others show the photoexcited regions enough long after the laser irradiation. (a) Irradiation with single 300 fs laser pulse of $2 \mu \mathrm{J}$. (b), (c) Irradiation with double $300 \mathrm{fs}$ laser pulse of $1 \mu \mathrm{J}$ and $1 \mu \mathrm{J}$.

( $>0.2 \mathrm{~s}$ ) the laser irradiation. The laser focal region at the center of the image became dark. This transmission change in the laser focal region suggests that laser-induced plasma had been generated by photoexcitation [6]. Although the transient change by photoexcitation was observed clearly just after the photoexcitation (Fig. 1(a), left), no permanent modification was observed (Fig. 1(a), middle) even after 5 shots (Fig. 1(a), right). In fact, ten-thousands shots of 100 fs laser pulses could not induce any permanent modification. With much higher pulse energy (>10 $\mu \mathrm{J})$, damage occurred on the surface of the diamond sample.

\subsection{Irradiation with double pulses-trains}

Figure 2 (b) and (c) show pump-probe microscope images after double-pulse irradiation with $300 \mathrm{fs}$ laser pulses and images long after laser irradiation (>0.2 s). The time of the pump-probe observation was $1 \mathrm{ps}$ after the arrival of the second pulse at the laser focal region. The energies of two laser pulses were both $1 \mu \mathrm{J}$. Although the total energy of laser pulses was the same as that of single-pulse irradiation, the dark region in the laser focal region was smaller than that of single-pulse irradiation. In addition, permanent modifications occurred by a single shot of double-pulse irradiation. After the second shot, the modification became much clearer. This significant change of permanent modification by the second shot suggests that the highly absorbing materials were generated by the first shot. Interestingly, the permanent modification depended on the pulse delay between double pulses $\left(\Delta t_{d}\right)$; the permanent modification of $\Delta t_{d}=10$ ps (Fig. 2 (c)) was larger than that of $\Delta t_{d}=1$ ps (Fig. 2 (b)).

Figure 3 (a) and (b) show transmission optical microscope images of the permanent modifications along the laser propagation direction. These modifications were induced by a single-shot of double-pulse irradiation with different $\Delta t_{d}$. The pulse durations for Fig. 3 (a) and 3 (b) were $200 \mathrm{fs}$ and $300 \mathrm{fs}$, respectively. In fact, no permanent modification appeared at the pulse duration of $100 \mathrm{fs}$ even by double-pulse irradiation with any $\Delta t_{d}$. At the pulse duration of 200 fs [Fig. 3 (a)], no permanent modification ap-

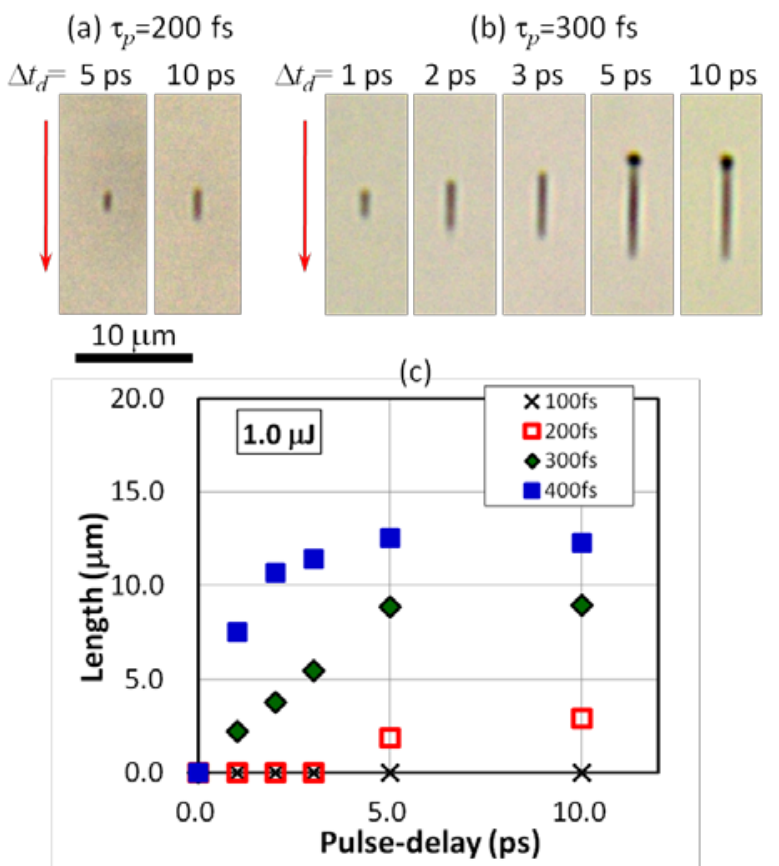

Fig. 3 (a), (b) Transmission optical microscope images of modifications inside diamond by double-pulse irradiation with $200 \mathrm{fs}$ and 300 fs laser pulses, respectively. The pulse energies of double pulses were equal, $1 \mu \mathrm{J}$. The time written above each image is the pulse delay between double pulses. The arrows show the incident direction of the laser pulse. (c) The longitudinal lengths of the modifications plotted against the pulse delay.

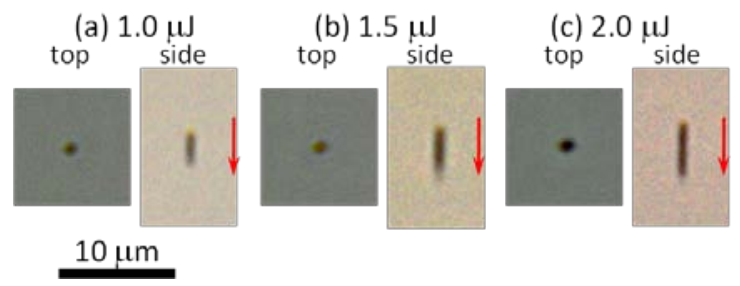

Fig. 4 Transmission optical microscope images of modifications inside a diamond by a single irradiation with a single 400 fs laser pulse. "Top" and "side" refer to observation parallel and perpendicular to the laser propagation direction, respectively. The arrows show the incident direction of the laser pulse.

peared for $\Delta t_{d} \leq 2 \mathrm{ps}$. In contrast, for a pulse duration of $300 \mathrm{fs}$, modifications were observed for all $\Delta t_{d}$ from 1 to 10 ps. The longitudinal lengths of the modifications for different pulse durations were plotted against $\Delta t_{d}$ in Fig. 3 (c). The longitudinal length increased with $\Delta t_{d}$ in the range of $1-10$ ps and with pulse duration.

In fact, we found that permanent modifications were able to be induced by single-pulse irradiation with a $400 \mathrm{fs}$ laser pulse. Figure 4 shows the optical microscope images of permanent modifications by single-pulse irradiation with a 400 fs laser pulse. The larger modification occurred at higher pulse energy in the range of $1-2 \mu \mathrm{J}$. On the other hand, when the pulse duration was 300 fs or shorter, no modification occurred even after 100 shots by single-pulse irradiation in our experiment. It is possible that photoexcitation by the longer $400 \mathrm{fs}$ laser pulse has a similar effect as double-pulse irradiation, considering that a 400 fs laser pulse has a similar effect on the photoexcitation process as 
double 300 fs pulses, because similar modification appeared by double $300 \mathrm{fs}$ pulses with the shortest $\Delta t_{\mathrm{d}}$.

\subsection{Transmission change after laser irradiation}

The dynamics after the laser irradiation was measured by the observation of the transmission change using a pumpprobe microscope to gain further insight into the doublepulse interaction mechanisms in diamond. Figure 5 shows pump-probe transmission images at different times after irradiation with single and double 300 fs-pulses. The transmission changes by single-pulse and double-pulse irradiations were essentially the same: the photoexcited region became darker at 1 ps after the photoexcitation, a bright spot appeared at the center of the photoexcited region in 100 ps-240 ps. In 160-240 ps, a wave grew gradually around the photoexcited region and propagated away from the photoexcited region (indicated by white arrows in the images). After the wave propagated away out of the images (after 1000 ps), the bright spot in the photoexcited region became weaker and almost disappeared at 6000 ps.

(a) Single pulse $(2 \mu \mathrm{J})$

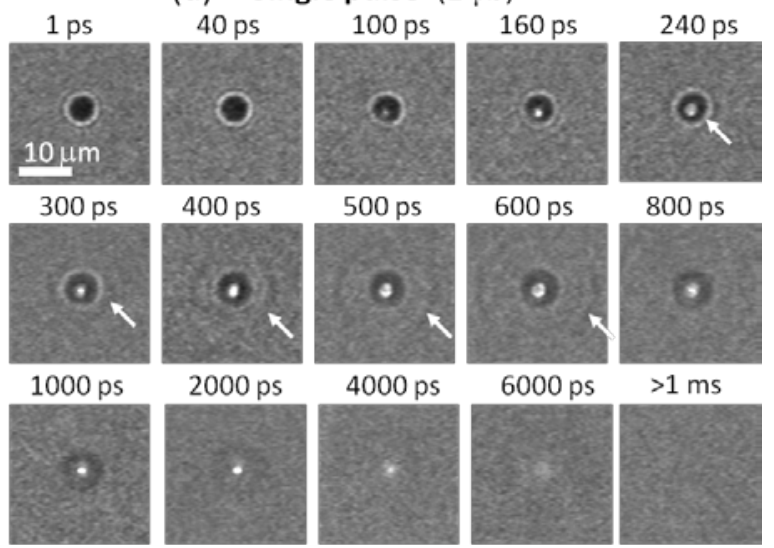

(b) Double pulses $\left(1 \mu \mathrm{J}+1 \mu \mathrm{J}, \Delta t_{d}=10 \mathrm{ps}\right)$
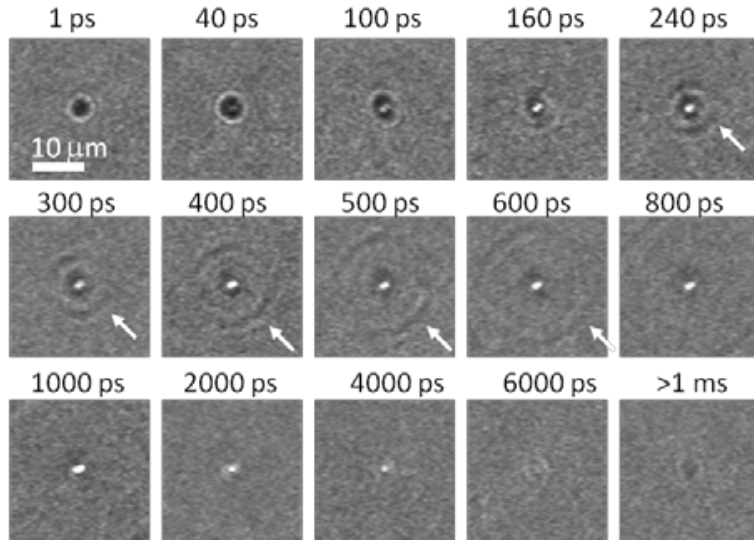

4000 ps
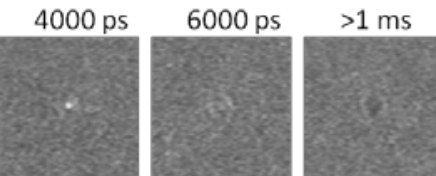

Fig. 5 Pump-probe microscope images in the photoexcited region inside diamond at different times after irradiation with 300 fs laser single and double pulses. (a) Irradiation with a single pulse of $2 \mu \mathrm{J}$. (b) Irradiation with double pulses of $1 \mu \mathrm{J}+1 \mu \mathrm{J}$ with $\Delta t_{d}=10 \mathrm{ps}$. In (b), the times shown above the images means the times after the photoexcitation by the second pulse. The white arrows in the images from 240 ps to 400 ps indicate stress waves.

Regardless of the similar transmission changes, permanent modification appeared only by the double-pulse irradiation (Images at $>1 \mathrm{~ms}$ in Fig. 5).
The appearance of the dark spot at 1 ps should be attributed to the laser-induced electron-hole plasma, which has been observed by Kononenko et al [6]. Although several researchers observed refractive index change in 20-30 ps after fs laser irradiation in diamond $[6,16]$, our observation did not show any clear change in the time scale.

The velocity of the propagating wave in 240 ps-800 ps was about $18 \mu \mathrm{m} / \mathrm{ns}$ in the upper direction, which is similar to the reported velocities of the longitudinal elastic wave in diamond $\left(\sim 1.7 \times 10^{4} \mathrm{~m} / \mathrm{s}\right.$ in [100] and $\sim 1.8 \times 10^{4} \mathrm{~m} / \mathrm{s}$ in [110]) [17]. Therefore, this wave should be attributed to a laser-induced stress wave.

The disappearance of the bright spot after 1000 ps should be attributed to the heat dissipation from the photoexcited region, because the bright spot decayed and became larger in the time-scale of the thermal diffusion in diamond, which had been observed by Kononenko et al [6].

The main difference between the observed transient optical transmission images in Fig. 5 (a) and 5 (b) is that the photoexcited region by double-pulse irradiation (dark region at $1 \mathrm{ps)}$ was about two times smaller than that of single-pulse irradiation. Regardless of different diameters of the photoexcited regions, the stress waves were observed clearly both after single- and double-pulse irradiation.

\subsection{Phase distribution change after laser irradiation}

To compare the amplitudes of the stress waves, the phase changes by the stress waves were observed by a pumpprobe microscope with a Michelson interferometer. Figure 6 (a) shows the interference pattern of the probe beam at 300 ps after single 300 fs laser pulse irradiation. The phase (a)
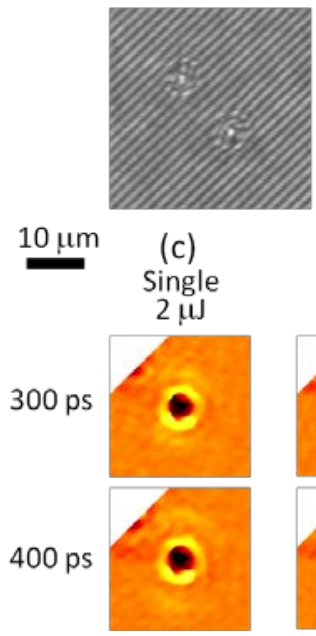

(b)

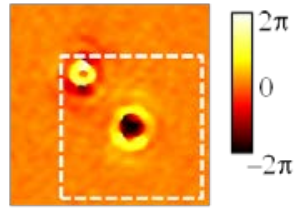

(d)

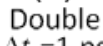

$\Delta t_{d}=1 \mathrm{ps}$

(e) Double $\Delta t_{d}=10 \mathrm{ps}$
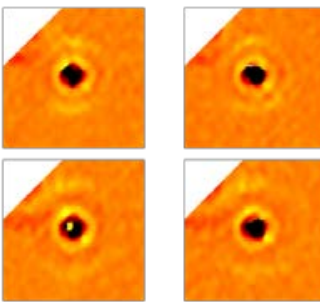

Fig. 6 (a) Interference pattern of the probe beam at 300 ps after single $300 \mathrm{fs}$ laser pulse irradiation on the CCD camera after a Michelson interferometer, and (b) phase distribution obtained by analysis of the interference pattern. (c)-(e) Phase distributions in diamond at 300 ps and 400 ps after the photoexcitation with the same total pulse energy. (c) Singlepulse irradiation by a $2 \mu \mathrm{J}, 300$ fs laser pulse. (d), (e) Double-pulse irradiation by two $1 \mu \mathrm{J}, 300$ fs laser pulses. The total pulse energy were the same in (c)-(e).

distribution was obtained by the analysis of the interference pattern based on the Fourier transform method [15]. The 
obtained phase distribution is shown in Fig. 6 (b). There are double-ring patterns in the phase distribution. This doublering pattern comes from the interference of two identical probe beams, which had been split in the Michelson interferometer (The principle is described in detail in Section 2.2.). Therefore, these phase distributions correspond to $\Delta \phi(x, y)-\Delta \phi(x+\Delta x, y+\Delta y)$, where $\Delta \phi(x, y)$ is the phase distribution change in the photoexcited region and $(\Delta x, \Delta y)$ is the displacement of one probe beam from the other one. Here, we focus the lower right phase distributions in the region enclosed by a white broken line in Fig. 6 (b).

The phase distributions at 300 ps and 400 ps after single and double-pulse irradiation are shown in Fig, 6 (c)-(e). The phase decreased in the central region (i.e. the photoexcited region) and positive phase change appeared at the periphery of the central region. At several micrometers from the central region, another smaller positive phase change can be observed. This positive phase change moved away from the photoexcited region from 300 ps to 400 ps, and the positions are the same as the stress wave observed in the transmission images of Fig. 5. Therefore, this smaller positive ring should be attributed to the laser-induced stress wave.

The radial phase distributions from the center of the photoexcited regions were shown in Fig. 7 (a) and (b). We can see the propagation of the positive phase peak by a stress wave (the positions are indicated by black broken lines) from 300 ps to $400 \mathrm{ps}$ at about $\sim 2 \times 10^{4} \mathrm{~m} / \mathrm{s}$. The phase change of the stress wave (the phase on the broken line) by double-pulse irradiation was almost same as that by singlepulse irradiation. On the other hand, the phase changes at the periphery of the photoexcited region (the peak indicated by an arrow in Fig. 7) by double-pulse irradiation were two times smaller than that by single-pulse irradiation.

\section{Discussion}

The important findings in this study are (i) permanent modification occurred by single-shot of double-pulse irradiation when the pulse duration was 200-400 fs, while no permanent modification occurred even after 5 shots of sin-
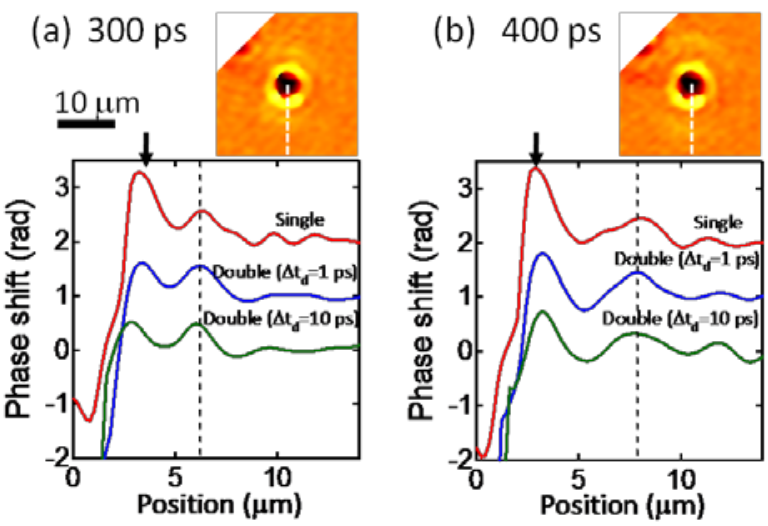

Fig. 7. (a), (b) Phase changes plotted against the distance from the center of the photoexcited region (along the white broken line in the images). The positions of the stress waves are indicated by vertical broken line. The phase distributions were offset for clarity.

gle-pulse irradiation when the pulse duration was 100-300 fs (Fig. 2); (ii) the longitudinal length of the modification by double-pulse irradiation became longer when the pulse delay was increased from 1 ps to 10 ps (Fig. 3 (c)); (iii) the phase change by a laser-induced stress wave was similar by single- and double-pulse irradiation (Fig. 7), while the width of the photoexcited region in the pump-probe images was smaller by double-pulse irradiation than that by singlepulse irradiation (Fig. 5); (iv) the phase change at the periphery of the photoexcited region by double-pulse irradiation was two times smaller than that by single pulseirradiation (Fig. 6, 7).

\subsection{Modification by double-pulse irradiation}

The enhancement of fs laser-induced permanent modification by double-pulse irradiation has been reported also in other materials [18-20]. For example, Sugioka et al showed that thermal energy by fs laser irradiation inside a glass become larger by double-pulse irradiation [17, 18]. For modifications in a single crystal, Mori et al. found that only double-pulse irradiation with infrared fs laser pulses can induce permanent modifications inside single crystalline silicon [19]. In these reports, the enhancement of the modification by double-pulse irradiation is attributed to electron-hole plasmas or self-trapped excitons (STEs) generated by the first laser pulse, because the plasma and STEs can absorb the second pulse efficiently.

According to the pump-probe study by Guizard et al., the generation of STEs was not observed after fs laser irradiation in diamond [21]. They explained that diamond is so rigid that an electron-hole pair cannot deform the crystal lattice to form a trapped level for STEs [7]. However, the situation in the photoexcited region inside diamond in our study is completely different from that in the experiment by Guizard et al. In the laser irradiation condition in our study, in which the laser intensity at the focus $\left(>1 \times 10^{13} \mathrm{~W} / \mathrm{cm}^{2}\right)$ was much higher than that in the experiment by Guizard et al. $\left(3 \times 10^{11}-1.5 \times 10^{12} \mathrm{~W} / \mathrm{cm}^{2}\right)$, the temperature in the photoexcited region just after the photoexcitation could be so high that the diamond lattice at high temperature can be deformed by electron-hole pairs, so STEs can be generated.

In our study, we found that the modification by doublepulse irradiation in diamond became larger at longer pulse in the range of $\Delta t_{\mathrm{d}}=1 \mathrm{ps}-10 \mathrm{ps}$ (Fig. 3). The larger modification at longer pulse delay suggests that some dynamics in the photoexcited state, such as the generation of STEs or thermal equilibrium between electrons and lattices, could occur in 1 ps-10 ps and that the photoexcitation of STEs or electron-hole plasmas in thermal equilibrium may be necessary for generation of permanent modification.

\subsection{Different dynamics between single- and double- pulse irradiation}

The main difference in dynamics between single- and double-pulse irradiation is the diameter of the photoexcited region in the transmission images (Fig. 5). The diameter of the photoexcited region by single-pulse irradiation were about two times larger than that by double-pulse irradiation with the same total pulse energies. The larger diameter of the photoexcitation by single-pulse irradiation should be attributed to the higher laser intensity at the focus, because the higher laser intensity at the focus causes larger nonlinear photoexcitation, and plasma defocusing, which results in photoexcitation in a larger volume [22]. In double-pulse irradiation, the diameter of the photoexcited region by the 
first laser pulse is smaller because of two times lower laser intensity. In addition, the phase change at the periphery of the photoexcited region by single-pulse irradiation was also two times larger than that by double-pulse irradiation (Fig. 6 and Fig. 7). If it is assumed that the phase change at the periphery of the photoexcited region is due to stress from the photoexcited region, the larger phase change by single pulse irradiation could be due to larger stress by the larger photoexcited volume.

On the other hand, the phase changes by stress waves were similar in single and double-pulse irradiation (Fig. 7). Because the amplitude of a stress wave becomes larger with increasing stress gradient in the origin of the stress wave (i.e. photoexcited region), the similar phase change by stress wave in single- and double-pulse irradiation suggests that the stress gradient by double-pulse irradiation should be larger than that by single pulse irradiation. Because the laser-induced plasma could be the origin of the stress wave, the smaller diameter of the photoexcited region, smaller stress and larger stress gradient in doublepulse irradiation suggests that high density plasma in a more limited volume can be generated by double-pulse irradiation.

Only from the observations in this paper, we cannot give any clear explanation of the mechanism of photoexcitation process and permanent modification in diamond by double-pulse irradiation. To elucidate how the photoexcitation by double-pulse irradiation induces the phase transformation in diamond, the plasma temperature, dynamics of STEs, lattice deformation in the photoexcited states and stress generation must be elucidated in more detail. In addition, the pump-probe observation perpendicular to the laser propagation direction should be necessary, because the longitudinal length of the permanent modification strongly depended on the pulse duration, pulse energy and pulse delays.

\section{Summary and Conclusion}

In this study, we showed that double-pulse irradiation with 200-400 fs laser pulses is effective to induce larger permanent modification inside diamond. The modification by double-pulse irradiation was larger at longer pulse duration in 100-400 fs and at longer pulse delay in 1-10 ps. The time-resolved observation of transmission images suggested that double-pulse irradiation can generate photoexcited electrons of high density in a smaller volume. However, further investigations are needed to explain why the double-pulse irradiation can more easily induce a phase transformation in diamond.

\section{Acknowledgments}

We would like to acknowledge the partial support of this work by CONCERT-JAPAN project of SICORProgram of JST, Japan, the FP7 DiamondFab CONCERT Japan project, DIAMANTE MIUR-SIR grant, and FemtoDiamante Cariplo ERC reinforcement grant, the Scientific and Technological Research Council of Turkey (TUBITAK) project number 114F312, and Japan Society for the Promotion of Science (JSPS) Grant-in-Aid for Scientific Research (C), No. 26410240.

\section{References}

[1] W. Pfaff, T. H. Taminiau, L. Robledo, H. Bernien1, M. Markham, D. J. Twitchen, and R. Hanson: Nature Phys., 9, (2013) 29. [2] L. Childress, R. Walsworth, and M. Lukin: Phys. Today, 67, (2014) 38.

[3] J. Burghoff, H. Hartung, S. Nolte, and A. Tünnermann: Appl. Phys. A, 86, (2007) 165.

[4] F. Chen, and J. R. V. de Aldana: Laser Photonics Rev., 8, (2014) 251.

[5] T. V. Kononenko, M. Meier, M. S. Komlenok,S. M. Pimenov, V. Romano,V. P. Pashinin, and V. I. Konov: Appl, Phys. A, 90, (2008) 645.

[6] V. V. Kononenko, E. V. Zavedeev, M. I. Latushko, and V. I. Konov: Laser Phys. Lett., 10, (2013) 036003.

[7] V. V. Kononenko, V. M. Gololobov, M. S. Komlenok, and V. I. Konov: Laser. Phys. Lett., 12, (2015) 096101.

[8] G. Conte, P. Allegrini, M. Pacilli, S. Salvatori, T. kononenko, A. Bolshakov, V. Ralchenko, and V. Konov: Nuc. Inst. Meth. Phys. Res. A, 799, (2015) 10.

[9] T. V. Kononenko, P. N. Dyachenko, and V. I. Konov: Opt. Lett., 39, (2014) 6962.

[10] A. Jesacher, and M. J. Booth: Opt. Express, 18, (2010) 21090. [11] B. Sun, P. S. Salter, and M. J. Booth: Appl. Phys. Lett. 105, (2014) 231105.

[12] M. Shimizu, Y. Shimotsuma, M. Sakakura, T. Yuasa, H. Homma, Y. Minowa, K. Tanaka, K. Miura, and K. Hirao: Opt. Express, 17, (2009) 46.

[13] N. Morita, Y. Shimotsuma, M. Nishi, M. Sakakura, K. Miura, and K. Hirao: Appl. Phys. Lett., 105, (2014) 201104.

[14] Y. Hayasaki, M. Isaka, A. Takita, and S. Juodkazis: Opt. Express, 19, ( 2011) 5725.

[15] M. Takeda, H. Ina, and S. Kobayashi: J. Opt. Soc. Am., 72, (1982) 156.

[16] M. Kozák, F. Trojánek, and P. Malý, New Journal of Physics, 17 (2015) 053027.

[17] S.-F. Wang, Y.-F. Hsu, J.-C. Pu, J. C. Sung, and L. G. Hwa: Mat. Chem. Phys. 85, (2004) 432.

[18] K. Sugioka, M. Iida, H. Takai, and K. Micorikawa: Opt. Lett. 36, (2011) 2734.

[19] S. Wu, D. Wu, J. Xu, Y. Hanada, R. Suganuma, H. Wang, T. Makimura, K. Sugioka, and K. Midorikawa, Opt. Express, 20 (2011) 28893.

[20] M. Mori, Y. Shimotsuma, T. Sei, M. Sakakura, K. Miura, and H. Udono: Phys. Status Solidi A, 212, (2015) 715.

[21] P. Martin, S. Guizard, P. Daguzan, G. Petite, P. D’Oliveira, P. Meynadier, and M. Perdrix: Phys. Rev. B 55, (1997) 5799.

[22] N.M. Bulgakova, V.P. Zhukov, Y.P. Meshcheryakov, Appl. Phys. B, 113 (2013) 437.

(Received: May 21, 2016, Accepted: September 11, 2016) 